\title{
Temperatura base para abertura de floretes e antocrono em gladíolo ${ }^{1}$
}

\author{
Natalia Teixeira Schwab ${ }^{2}$, Nereu Augusto Streck ${ }^{2}$, Lilian Osmari Uhlmann ${ }^{2}$, Bruna San Martin Rolin Ribeiro ${ }^{2}$, \\ Camila Coelho Becker², Josana Andreia Langner ${ }^{2}$
}

\section{RESUMO}

O gladíolo é uma flor de corte de grande importância nos mercados nacional e mundial. A haste floral, do tipo espiga, é composta por floretes que apresentam abertura acropetal. O conhecimento da velocidade de abertura desses floretes, representada pelo antocrono, pode auxiliar no planejamento das atividades de colheita e comercialização. Os objetivos deste trabalho foram: estimar a temperatura base para abertura de floretes em gladíolo e determinar o antocrono, com a unidade de tempo representada pela soma térmica. Foram conduzidos dois experimentos de campo, em Santa Maria, RS, durante dois anos (2011 a 2013), sendo cada experimento composto por 12 datas de plantio e três cultivares. A soma térmica diária (STd) foi calculada pela diferença entre a temperatura média e a temperatura base (Tb) e, a soma térmica acumulada, pelo somatório das STd. Para a estimativa Tb para abertura de floretes, utilizaram-se os dados do número de floretes abertos (NFA), coletados nos experimentos. Conclui-se que Tb para abertura de floretes em gladíolo é $6^{\circ} \mathrm{C}$ e que o antocrono é, em média, $14,7^{\circ} \mathrm{C}$ dia por florete, mas varia com a disponibilidade de radiação solar durante o florescimento da espiga.

Palavras-chave: Gladiolus x grandiflorus; floricultura; temperatura basal; soma térmica; radiação solar.

\begin{abstract}
Base temperature for florets opening and anthochron in gladiolus

Gladiolus is an important cut flower in national and international markets. The floral stem, a spike, is composed of florets that have acropetally opening. Knowledge on the speed opening of florets, represented by the anthochron, can assist in planning the harvesting and marketing activities. The objectives of this study were estimate the base temperature for florets opening in gladiolus and to determine the anthochron, with the time unit represented by the thermal time. Two field experiments during two years (2011-2013), each experiment composed of 12 planting dates and three cultivars, were conducted in Santa Maria, RS, Brazil. The daily thermal (STd) was calculated as the difference between the daily mean temperature and the base temperature $(\mathrm{Tb})$, and the accumulated daily thermal was obtained by the sum of STd. To estimate the Tb to florets opening, we used the number of open florets (NFA) collected in the experiments. We concluded that the $\mathrm{Tb}$ for florets opening in gladiolus is $6^{\circ} \mathrm{C}$ and the anthochron is, on the average, $14,7^{\circ} \mathrm{C}$ day per floret, but the anthochron varies according to the solar radiation during the spike flowering period.
\end{abstract}

Key words: Gladiolus x grandiflorus, floriculture, base temperature, daily thermal, solar radiation.

\footnotetext{
Submetido em 17/12/2015 e aprovado em 04/10/2017.

1 Este trabalho é parte da tese de doutorado da primeira autora.

${ }^{2}$ Universidade Federal de Santa Maria, Departamento de Fitotecnia, Santa Maria, Rio Grande do Sul, Brasil. natalia_schwab@hotmail.com; nstreck2@yahoo.com.br; liliuhlmann@yahoo.com.br; brunasanmartinrolim@gmail.com; camilabecker07@hotmail.com; langnerja@gmail.com

*Autora para correspondência: natalia_schwab@hotmail.com
} 


\section{INTRODUÇÃO}

Gladiolus x grandiflorus Hort., popularmente conhecido como gladíolo ou palma-de-Santa-Rita, é uma planta herbácea, pertencente à família Iridaceae, obtida pela hibridação entre diversas espécies nativas do sul do continente africano e do mediterrâneo. É uma planta heliófila, propagada vegetativamente por meio de bulbos. Pode ser cultivada ao longo de todo o ano, exceto em regiões que apresentem períodos com geadas. A inflorescência é do tipo espiga e se destaca como importante flor de corte no mercado mundial (Ahmad et al., 2008; Ahmad et al., 2011; Singh et al., 2012). No Brasil, apresenta pico de comercialização na época de Finados (Schwab et al., 2015a), sendo uma ótima opção para exploração na agricultura familiar.

Para construir a logística de colheita e distribuição das hastes de gladíolo, bem como conhecer a vida-deprateleira das hastes florais, é necessário que se conheça a velocidade de abertura dos floretes em uma haste. Para quantificar esta velocidade, foi proposto por Schwab et al. (2014) o termo antocrono, que representa o intervalo de tempo de abertura entre flores sucessivas em inflorescências, com unidade tempo por flor (exemplo: dias/flor). Com a unidade de tempo em dias, o antocrono para gladíolo variou com a época de plantio em ambiente subtropical e apresentou relação negativa com a temperatura do ar (Schwab et al., 2014), indicando a possibilidade de utilizar a soma térmica como unidade de tempo para reduzir ou eliminar o efeito de época sobre o antocrono. No entanto, para que o antocrono possa ser estimado em ${ }^{\circ} \mathrm{C}$ dia por florete é necessário que seja conhecida a temperatura base $(\mathrm{Tb})$ para a abertura de floretes em gladíolo.

A temperatura base é definida como a temperatura abaixo da qual o desenvolvimento não ocorre ou, se ocorrer, é a uma taxa desprezível (Arnold, 1960). Na literatura, são encontradas temperatura-base para inúmeras espécies, incluindo plantas daninhas como papuã (Brachiaria plantaginea (Link) Hitchc) e malmequer-do-campo (Aspilia montevidensis (Spreng.)); e plantas cultivadas, como trigo (Triticum aestivum), triticale (Tritico secale Wittmack), milho (Zea mays L.). Para o desenvolvimento floral do gladíolo, no entanto, não foi encontrado nenhum valor de temperatura base. A hipótese é que o antocrono, dado em ${ }^{\circ} \mathrm{C}$ por dia por florete, não varia com a época de plantio.

Os objetivos deste trabalho, foram estimar a temperatura base para abertura de floretes em gladíolo e determinar o antocrono com a unidade de tempo representada pela soma térmica.

\section{MATERIAL E MÉTODOS}

Foram conduzidos dois experimentos de campo, durante dois anos (agosto de 2011 a julho de 2013), cada experimento com 12 datas de plantio (uma por mês), em Santa Maria, RS (29 43' S, 53 43' O, em altitude de 95m). Em cada experimento, o delineamento experimental foi o de blocos ao acaso, em esquema bifatorial, com quatro repetições, sendo cada repetição uma parcela de dez plantas, em que seis delas foram marcadas (área útil) e as demais formaram a bordadura. No Experimento 1, o fator " $A$ " foi o cultivar, com três níveis (Peter Pears, Rose Friendship e Jester, e, no Experimento 2, Amsterdã, Rose Friendship e Jester). Os cultivares selecionados apresentavam diferentes durações do ciclo de desenvolvimento (precoce, médio e tardio, respectivamente). O fator "B" foi a data de plantio, com 12 níveis. No Experimento 1, as datas foram: 05/08/2011;02/09/2011;03/10/2011;01/11/2011;01/12/ 2011;04/01/2012;01/02/2012;07/03/2012;02/04/2012;02/ 05/2012; 01/06/2012 e 02/07/2012 e, no Experimento 2, 02/ 08/2012; 03/09/2012;04/10/2012;01/11/2012;03/12/2012; 04/01/2013; 01/02/2013; 01/03/2013; 01/04/2013; 01/05/ 2013; 03/06/2013 e 02/07/2013.

Em cada data de plantio, foram utilizados bulbos comerciais de gladíolo, com circunferência variando entre 14 e $16 \mathrm{~cm}$, os quais foram plantados em canteiros de 1 metro de largura, 17 metros de comprimento e $10 \mathrm{~cm}$ de altura, contendo duas linhas pareadas espaçadas em 40 $\mathrm{cm}$, e $20 \mathrm{~cm}$ de espaçamento entre plantas na linha. Os blocos foram demarcados nos canteiros e distanciados de $1 \mathrm{~m}$ entre si. Cada data de plantio constituía um canteiro. Quando as anteras estavam visíveis, o florete foi considerado aberto, estádio R3 da escala de Schwab et al. (2015b). A partir do dia em que o primeiro florete foi considerado aberto passou-se a contar duas vezes por semana o número de floretes abertos na haste floral das plantas marcadas.

A soma térmica diária (STd, ${ }^{\circ} \mathrm{C}$ dia) foi calculada segundo (McMaster \& Wilhelm, 1997):

$\mathrm{STd}=(\mathrm{Tmed}-\mathrm{Tb}) .1 \mathrm{dia}$

em que Tmed é a temperatura média diária do $\operatorname{ar}\left({ }^{\circ} \mathrm{C}\right)$ e $\mathrm{Tb}$ é a temperatura base $\left({ }^{\circ} \mathrm{C}\right)$. Esse método foi escolhido por ser simples e de acurácia suficiente para a finalidade deste trabalho. A soma térmica acumulada $\left(\mathrm{STa},{ }^{\circ} \mathrm{C}\right.$ dia) foi obtida pelo somatório das STd, ou seja:

$\mathrm{STa}=\Sigma \mathrm{STd}$

A Tmed foi calculada pela média aritmética entre a temperatura mínima e máxima diária medida na Estação Convencional do Instituto Nacional de Meteorologia (INMET), localizada a aproximadamente $150 \mathrm{~m}$ do experimento.

Para a estimativa da temperatura base $(\mathrm{Tb})$ para abertura de floretes, utilizaram-se os dados do número de floretes abertos (NFA), coletados em sete datas de plantio no experimento $1(05 / 08 / 2011 ; 02 / 09 / 2011 ; 03 / 10 / 2011$; 
02/04/2012; 02/05/2012; 01/06/2012 e 02/07/2012) e no experimento $2(02 / 08 / 2012 ; 03 / 09 / 2012 ; 04 / 10 / 2012 ; 01 / 04 /$ 2013; 01/05/2013; 03/06/2013 e 02/07/2013). Essas datas foram selecionadas por terem a abertura dos floretes nos meses com temperaturas amenas e, assim, possivelmente, mais próximas da $\mathrm{Tb}$. Para a estimativa da $\mathrm{Tb}$, foi utilizada a metodologia do menor quadrado médio do erro (QME) da regressão entre o NFA e a STa, a partir da emergência (Wutke et al., 2000; Sinclair et al., 2004). Foram estimadas equações de regressão linear simples para os valores do NFA médio do gladíolo, em cada data de plantio, em função da STa, utilizando-se vários valores de Tb, que variaram de zero a $21^{\circ} \mathrm{C}$, com incremento de $0,5^{\circ} \mathrm{C}$. O valor de $\mathrm{Tb}$ foi considerado como aquele com o menor QME (Sinclair et al., 2004).

Para cada repetição de seis plantas, foi feita uma regressão linear simples entre o número de floretes abertos, acumuladas na haste floral (NFA), e a soma térmica acumulada (STa) a partir da data em que os três floretes basais da espiga mostravam a cor da corola (estádio R2 da escala de Schwab et al., 2015b), utilizando-se a Tb estimada para abertura de floretes. O antocrono foi estimado como o inverso do coeficiente angular da regressão linear entre NFA e STa (Schwab et al., 2014). Os dados de antocrono foram submetidos à análise de variância e à comparação de médias, pelo teste de Tukey, a 5\%, considerando-se um bifatorial em cada experimento.

Também foi realizada uma regressão linear simples (com nível de confiança de 95\%), para cada repetição de seis plantas, entre o antocrono $\left({ }^{\circ} \mathrm{C}\right.$ dia) calculado para cada data de plantio e cultivar versus a temperatura média $\left({ }^{\circ} \mathrm{C}\right)$ e versus a radiação solar global acumulada $\left(\mathrm{MJ} \mathrm{m}^{-2}\right)$, ocorrente no período de florescimento da cultura, entre o estádio R2 (primeiros floretes na base da haste mostrando a cor da pétala) até o estádio R5 (abertura do último florete da haste) da escala de Schwab et al. (2015b).

\section{RESULTADOS E DISCUSSÃO}

Observou-se variação da $\mathrm{Tb}$, estimada em função da data de plantio e do cultivar e, em algumas datas de plantio não foi possível estimar-se a $\mathrm{Tb}$, pois o método não convergiu para um mínimo QME (Tabela 1). Essa variação também foi observada na estimativa da $\mathrm{Tb}$ para emissão de nós em melancia (Lucas et al., 2012) e em batata-doce (Erpen et al., 2013). Em função da variação, optou-se por fazer a média das épocas e cultivares $\left(\mathrm{Tb}=5,8^{\circ} \mathrm{C} \mathrm{H}\right.$ " 6,0 ${ }^{\circ} \mathrm{C}$ ), como foi feito, segundo Lucas et al. (2012), para melancia e, segundo Erpen et al. (2013), para a batata-doce.

$\mathrm{O}$ valor de $\mathrm{Tb}$ igual a $6^{\circ} \mathrm{C}$ é próximo à temperatura de estocagem para flores de corte em geral, ou seja, um indicativo da temperatura base já que o objetivo da estocagem à frio é paralisar ou retardar o processo de abertura das flores. Nowak et al. (1991) indicam que para flores de corte de origem subtropical, como o gladíolo, a temperatura ótima de estocagem varia de 4 a $7^{\circ} \mathrm{C}$, enquanto, para flores de corte de origem tropical, a temperatura ótima de estocagem está entre 7 e $15^{\circ} \mathrm{C}$. (Burg (2004) afirma que hastes de gladíolo devem ser estocadas a temperaturas de 2 a $5{ }^{\circ} \mathrm{C}$, a fim de retardar a abertura de floretes e prolongar a vida-de-prateleira.

Comparando-se a $\mathrm{Tb}$ estimada neste trabalho com as temperaturas-base na fase reprodutiva de outras culturas agrícolas, nota-se que o gladíolo é mais tolerante a baixas temperaturas. Por exemplo, a Tb durante a fase reprodutiva é $15^{\circ} \mathrm{C} \mathrm{em}$ arroz (Streck et al., 2011), $8^{\circ} \mathrm{C} \mathrm{em}$ milho (Streck et al., 2008) e $8^{\circ} \mathrm{C}$ em trigo (Streck et al., 2003). Esta maior tolerância do gladíolo às baixas temperaturas confere à cultura boa adaptação a regiões de clima subtropical, como, por exemplo, o sul do Brasil, onde, potencialmente, a espécie pode ser cultivada na primavera, no verão e no outono.

Com a temperatura base de $6,0^{\circ} \mathrm{C}$, foi calculada a soma térmica acumulada (STa), a partir dos primeiros três botões mostrando a cor da corola. Para o Experimento $1 \mathrm{e}$ para o Experimento 2, observou-se interação significativa entre datas de plantio e os cultivares, para a variável antocrono e, assim, a análise estatística foi desdobrada (Tabela 2). No Experimento 1, os cultivares apresentaram diferença estatisticamente significativa para os valores de antocrono calculados, apenas para os plantios realizados em 02/09/2011, 01/11/2011, 01/02/2012 e 02/07/2012. Já, no Experimento 2, os cultivares apresentaram diferença estatística nos valores de antocrono no plantio em 01/ 11/2012.

Quanto aos efeitos das datas de plantio sobre o antocrono (Tabela 2), no Experimento 1, os menores valores foram alcançados nos plantios de 05/08/2011, para o cultivar Peter Pears, e de 07/03/2012, para os cultivares Jester e Rose Friendship. Já no Experimento 2, os menores valores de antocrono foram nos plantios de 01/02/ 2013 e 01/03/2013, para o cultivar Amsterdã; de 01/04/2013, para o cultivar Jester; e 01/03/2013, 01/05/2013 e 03/06/ 2013, para o cultivar Rose Friendship. A média dos valores de antocrono obtidos nos meses de outono e inverno (abril, maio, junho, julho, agosto e setembro) e primavera e verão (outubro, novembro, dezembro, janeiro, fevereiro e março), para os dois experimentos, é $14,0^{\circ} \mathrm{C}$ dia por florete e $15,4^{\circ} \mathrm{C}$ dia por florete, respectivamente, ou seja, diferença de apenas $1,4^{\circ} \mathrm{C}$ dia por florete.

$\mathrm{Na}$ Figura 1, é apresentada a relação entre antocrono e temperatura média do ar, entre os estádios R2 e R5, nos dois experimentos de campo, cada um com 12 datas de plantio e os cultivares Peter Pears, Rose Friendship e Jester, no Experimento 1, e Amsterdã, Rose Friendship e Jester, no Experimento 2. A relação linear positiva (coefici- 
Tabela 1: Temperatura base para a abertura de floretes em gladíolo, estimada a partir de dados obtidos em dois experimentos de campo em Santa Maria, RS (Experimento 1, com plantios em 05/08/2011; 02/09/2011; 03/10/2011; 02/04/2012; 02/05/2012; 01/06/2012 e 02/ 07/2012 e Experimento 2, com plantios em 02/08/2012; 03/09/2012; 04/10/2012; 01/04/2013; 01/05/2013; 03/06/2013 e 02/07/2013)

\begin{tabular}{lcccccc}
\hline & \multicolumn{5}{c}{ Experimento/Cultivar } \\
\cline { 2 - 7 } Mês de Plantio & $\mathbf{1}$ & $\mathbf{2}$ & $\mathbf{1}$ & $\mathbf{2}$ & $\mathbf{1}$ & $\mathbf{2}$ \\
\hline AGO & Jester & Jester & RoseFriendship & RoseFriendship & Peter Pears & Amsterdã \\
SET & 0 & 0 & - & 0 & 17 & 0 \\
OUT & 0 & 0 & 4,5 & 19,5 & 17,5 & 0 \\
ABR & 10,5 & - & 0 & - & - & 0 \\
MAI & $-*$ & 3 & - & 0 & - & - \\
JUN & 0 & 2 & 0,5 & 7,5 & 0 & - \\
JUL & - & 0 & 20 & 0 & - & 17,5 \\
\hline Média & 17,5 & 0 & $5,8 \approx 6,0$ & &
\end{tabular}

* Não foi possível estimar.

Tabela 2: Antocrono $\left({ }^{\circ} \mathrm{C}\right.$ dia/florete) para quatro cultivares de gladíolo (Peter Pears, Jester, Rose Friendship e Amsterdã), em dois experimentos (Experimento 1: 2011/12 e Experimento 2: 2012/13)

\begin{tabular}{|c|c|c|c|}
\hline \multirow{3}{*}{ Data de plantio } & \multicolumn{3}{|c|}{ Experimento 1} \\
\hline & \multicolumn{3}{|c|}{ Cultivar } \\
\hline & Peter Pears & Jester & Rose Friendship \\
\hline 05/08/2011 & $10,2 \mathrm{~B} \mathrm{~d}^{*}$ & $15,5 \mathrm{Aab}$ & - \\
\hline 02/09/2011 & $14,9 \mathrm{Bbc}$ & $12,2 \mathrm{Cbc}$ & $17,6 \mathrm{~A} \mathrm{abc}$ \\
\hline 03/10/2011 & $15,4 \mathrm{Aab}$ & $14,0 \mathrm{Abc}$ & $14,9 \mathrm{Abc}$ \\
\hline $01 / 11 / 2011$ & 16,9 Bab & $22,2 \mathrm{AB}$ a & $26,0 \mathrm{Aa}$ \\
\hline 01/12/2011 & 18,6 Aab & $13,7 \mathrm{Abc}$ & $15,7 \mathrm{Abc}$ \\
\hline 04/01/2012 & $17,9 \mathrm{Aab}$ & $17,6 \mathrm{Aab}$ & $14,5 \mathrm{Abc}$ \\
\hline $01 / 02 / 2012$ & $19,3 \mathrm{Aa}$ & $11,0 \mathrm{Bbc}$ & - \\
\hline 07/03/2012 & $-* *$ & $7,6 \mathrm{Ac}$ & $9,7 \mathrm{Ac}$ \\
\hline 02/04/2012 & - & - & - \\
\hline 02/05/2012 & - & $17,6 \mathrm{Aab}$ & $15,9 \mathrm{Abc}$ \\
\hline 01/06/2012 & 10,9 Acd & $12,8 \mathrm{Abc}$ & $18,1 \mathrm{Abc}$ \\
\hline 02/07/2012 & - & $10,3 \mathrm{Bbc}$ & 20,1 Aab \\
\hline \multirow[t]{2}{*}{$\mathrm{CV}(\%)$} & \multicolumn{3}{|c|}{16,7} \\
\hline & \multicolumn{3}{|c|}{ Experimento 2} \\
\hline \multirow[t]{2}{*}{ Data de plantio } & \multicolumn{3}{|c|}{ Cultivar } \\
\hline & Amsterdã & Jester & Rose Friendship \\
\hline 02/08/2012 & $12,8 \mathrm{Aab}$ & $13,3 \mathrm{Aab}$ & $16,1 \mathrm{~A} a b c$ \\
\hline 03/09/2012 & 15,1 Aab & 14,2 Aab & $16,5 \mathrm{~A} a b c$ \\
\hline 04/10/2012 & 16,7 Aab & $15,9 \mathrm{Aa}$ & $18,5 \mathrm{Aab}$ \\
\hline 01/11/2012 & $12,1 \mathrm{~B} \mathrm{ab}$ & - & $20,4 \mathrm{~A}$ a \\
\hline $03 / 12 / 2012$ & - & - & . \\
\hline 04/01/2013 & $19,0 \mathrm{Aa}$ & 14,7 Aab & $14,8 \mathrm{~A}$ abc \\
\hline 01/02/2013 & $11,8 \mathrm{~A} \mathrm{~b}$ & $14,3 \mathrm{Aab}$ & $12,8 \mathrm{Abc}$ \\
\hline 01/03/2013 & $10,9 \mathrm{~A} \mathrm{~b}$ & $11,1 \mathrm{Aab}$ & $10,5 \mathrm{~A} \mathrm{c}$ \\
\hline 01/04/2013 & - & $9,0 \mathrm{~b}$ & - \\
\hline 01/05/2013 & - & 15,2 Aab & $10,6 \mathrm{Ac}$ \\
\hline 03/06/2013 & 13,1 Aab & 13,7 Aab & $11,3 \mathrm{~A} \mathrm{c}$ \\
\hline 02/07/2013 & 14,1 Aab & 14,6 Aab & $13,9 \mathrm{~A} \mathrm{bc}$ \\
\hline $\mathrm{CV}(\%)$ & & 16,5 & \\
\hline
\end{tabular}

*Médias seguidas pela mesma letra maiúscula, na linha, e minúscula, na coluna, não diferem pelo teste de Tukey a 5\%. ** Parcelas perdidas.

Rev. Ceres, Viçosa, v. 64, n.6, p. 557-560, nov/dez, 2017 
ente angular maior que zero) entre antocrono $\left({ }^{\circ} \mathrm{C}\right.$ dia/ florete) e temperatura média do ar poderia ser uma possível causa do efeito de época de plantio sobre o antocrono (Tabela 2). No entanto, como o efeito da temperatura so- bre a velocidade de abertura de floretes já está contemplada na unidade de tempo ${ }^{\circ} \mathrm{C}$ dia (Equação 1), outro fator que pode ser responsável pela variação do antocrono com a época de plantio é a radiação solar.

(A)

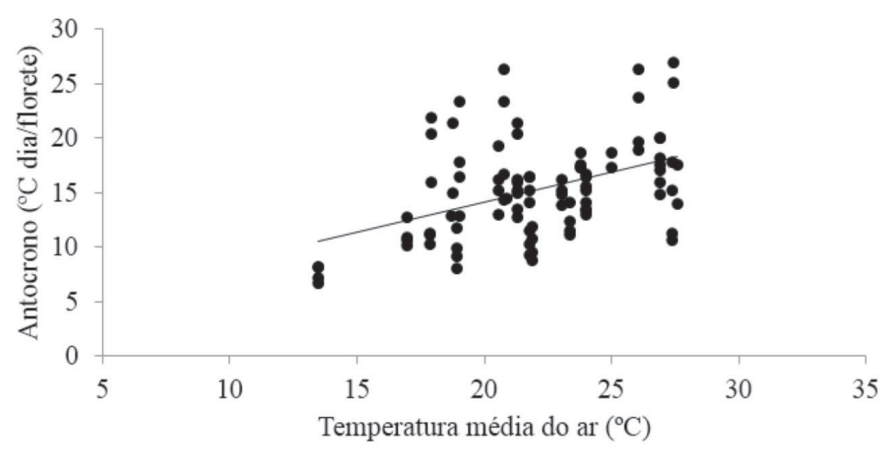

(B)

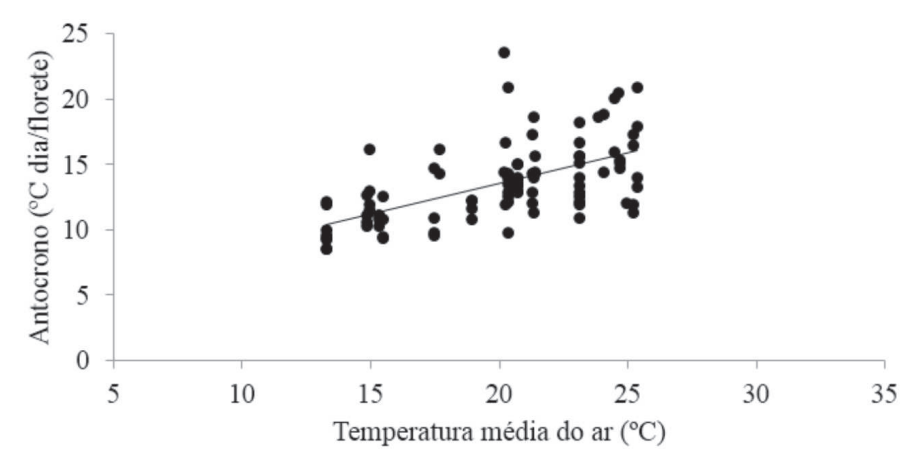

Figura 1: Relação entre antocrono $\left({ }^{\circ} \mathrm{C}\right.$ dia/florete) e temperatura média do ar $\left({ }^{\circ} \mathrm{C}\right)$, no Experimento 1 (A), com os cultivares Peter Pears, Rose Friendship e Jester, e no Experimento 2 (B), com os cultivares Amsterdã, Rose Friendship e Jester. Cada experimento reúne dados coletados em 12 datas de plantio e três cultivares.

(A)

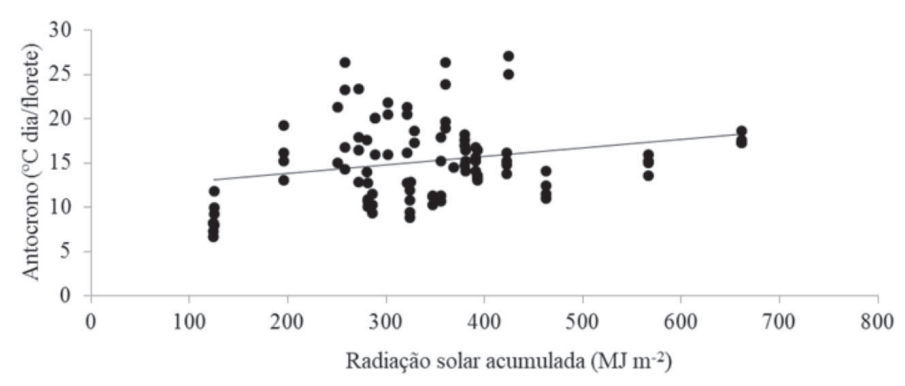

(B)

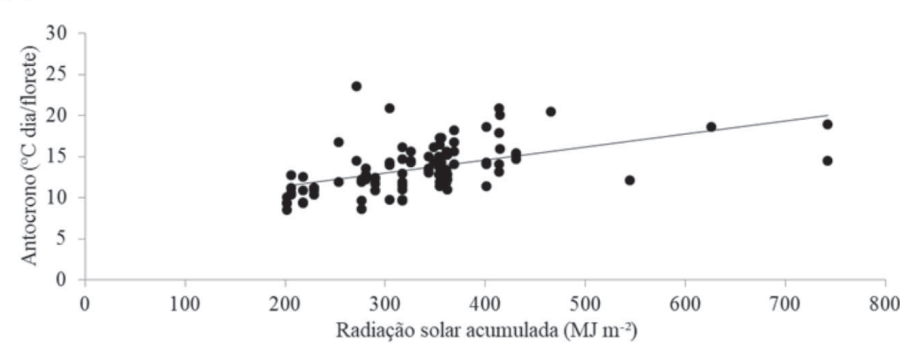

Figura 2: Relação entre antocrono $\left({ }^{\circ} \mathrm{C}\right.$ dia/florete) e radiação solar acumulada ( $\left.\mathrm{MJ} \mathrm{m}^{-2}\right)$, no experimento 1 (A), com os cultivares Peter Pears, Rose Friendship e Jester, e no experimento 2 (B), com os cultivares Amsterdã, Rose Friendship e Jester. Cada experimento reúne dados coletados em 12 datas de plantio e três cultivares. 
Na Figura 2, é apresentada a relação entre antocrono e a radiação solar acumulada entre os estádios R2 e R5, nos dois experimentos de campo. A relação positiva (coeficiente angular maior que zero) indica que a disponibilidade de energia fotossintética afeta a velocidade de abertura dos floretes, fato que fisiologicamente pode ser interpretado como a necessidade da planta de produzir fotoassimilados em alta quantidade para que os floretes possam completar seu desenvolvimento mais rapidamente. O processo de desenvolvimento até a abertura dos floretes demanda grande quantidade de reservas, por isso alta radiação solar durante o florescimento deve auxiliar na velocidade do processo, já que as folhas da planta de gladíolo estão verdes e fotossinteticamente ativas. Além disso, durante o florescimento tem início também o crescimento intenso do cormo filho e dos cormilhos, ou seja, quanto maior a radiação disponível, mais reservas estarão disponíveis para ambos os drenos.

\section{CONCLUSÕES}

A partir dos dados observados, conclui-se que: a temperatura base para abertura de floretes em gladíolo é $6^{\circ} \mathrm{C}$; o antocrono para gladíolo é, em média, $14,7^{\circ} \mathrm{C}$ dia por florete, mas varia com a disponibilidade de radiação durante o florescimento (estádios R2 e R5).

\section{REFERÊNCIAS}

Ahmad I, Khattak AM, Ara N \& Amin NU (2011) Effect of planting dates on the growth of gladiolus corms in Peshawar. Sarhad Journal of Agriculture, 27:195-199.

Ahmad T, Ahmad I \& Qasim M (2008) Present status and future prospects of gladiolus cultivation in Punjab, Pakistan. Journal of Tekirdag Agricultural Faculty, 5:227-238.

Arnold CY (1960) Maximum-minimum temperatures as a basis for computing heat units. Journal of the American Society for Horticultural Sciences, 76:682-692.

Burg SP (2004) Postharvest physiology and hypobaric storage of fresh produce. Florida, CABI Publishing. 670p.

Erpen L, Streck NA, Uhlmann LO, Langner JA, Winck JE \& Gabriel LF (2013) Estimativa das temperaturas cardinais e modelagem do desenvolvimento vegetativo em batata-doce. Revista Brasileira de Engenharia Agrícola e Ambiental, 17:12301238 .

Lucas DDP, Streck NA, Bortoluzzi MP, Trentin R \& Maldaner IC (2012) Temperatura base para emissão de nós e plastocrono de plantas de melancia. Revista Ciência Agronômica, 4:288-292.

McMaster GS \& Wilhelm WW (1997) Growing degree-days: one equation, two interpretations. Agricultural and Forest Meteorology, 87:291-300.

Nowak J, Goszczunska DM \& Rudnicki RM (1991) Storage of cut flowers and ornamental plants: present status and future prospects. Postharvest News and Information, 2:255-260.

Schwab NT, Streck NA, Becker CC, Langner JA, Uhlmann LO \& Ribeiro BSMR (2015b) A phenological scale for the development of Gladiolus. Annals of Applied Biology, 166:496-507.
Schwab NT, Streck NA, Langner JA, Ribeiro BSMR, Uhlmann LO \& Becker CC (2014) Aplicabilidade do termo antocrono para representar a velocidade de abertura de flores em inflorescência. Pesquisa Agropecuária Brasileira, 49:657-664.

Schwab NT, Streck NA, Ribeiro BSMR, Becker CC, Langner JA, Uhlmann LO \& Ribas GG (2015a) Parâmetros quantitativos de hastes florais de gladíolo conforme a data de plantio em ambiente subtropical. Pesquisa Agropecuária Brasileira, 50:902-911.

Sinclair TR, Gilbert RA, Perdomo RE, Shine Jr JM, Powell G \& Montes G (2004) Sugar cane leaf area development under field conditions in Florida, USA. Field Crops Research, 88:171-178.

Singh JP, Kumar K \& Katiyar PN (2012) Effect of zinc, iron and copper on yield parameters of gladiolus. HortFlora Research Spectrum, 1:64-68.

Streck NA, Lago I, Gabriel LF \& Samboranha FK (2008) Simulating maize phenology as a function of air temperature with a linear and a non-linear model. Pesquisa Agropecuária Brasileira, 43:449-455.

Streck NA, Lago I, Oliveira FB, Heldwein AB, Avila LA \& Bosco LC (2011) Modeling the development of cultivated rice and weedy red rice. Transactions of the ASAE, 54:371-384.

Streck NA, Weiss A, Xue Q \& Baenziger PS (2003) Improving predictions of developmental stages in winter wheat: A modified Wang and Engel model. Agricultural and Forest Meteorology, 115:139-150.

Wutke EB, Brunini O, Barbano MT, Castro JL, Gallo PB, Kanthack RAD, Martins ALM, Pereira JCVNA, Bortoleto N, Paulo EM, Sakai M, Saes LA, Ambrosano EJ, Carbonell SAM \& Silveira LCP (2000) Estimativa de temperatura base e graus-dia para feijoeiro nas diferentes fases fenológicas. Revista Brasileira de Agrometeorologia, 8:55-61. 